\title{
A review of housing-first strategies in reducing rates of substance use amongst people experiencing homelessness
}

\author{
Charles Yin, Stuart Danby \\ Faculty Reviewer: Abe Oudshoorn, RN, PhD (Arthur Labatt Family School of Nursing)
}

\section{ABSTRACT}

Substance use is a serious and prevalent health challenge among people who are homeless. The rate of alcohol use is 6 to 7 times more common in people experiencing homelessness than the general population, while approximately a quarter of all homeless individuals exhibit disordered use of one or more substances other than alcohol. Substance use is recognized as a significant risk factor for becoming homeless and is thought to complicate rehousing efforts and contribute to decreased adherence to rehabilitation programs that were and continue to be traditionally a requirement of rehousing. While studies have largely shown that housing-first strategies result in increased rates of retention in permanent housing compared to more established treatment-first strategies, it is less established whether housing-first strategies are equally successful among those homeless individuals with substance use challenges. In this review, we examine the available evidence on the efficacy of housing-first strategies in rehousing individuals with substance use challenges and in reducing the rates of substance use among people experiencing homelessness. We conclude that while housing-first strategies have not been shown to reduce rates of substance use compared to treatment-first strategies, both types of programs result in a comparable level of decrease in substance use rates despite treatment-first strategies mandating rehabilitation prior to rehousing. Finally, we provide a number of guidelines for an interdisciplinary approach to rehousing homeless individuals with substance use disorders through a housing-first strategy.

\section{INTRODUCTION}

Homelessness is a significant and growing problem in Canada. Homeless individuals are at greater risk of developing health problems and have significantly higher rates of early morbidity and mortality, all while having fewer resources and avenues to access necessary medical care. ${ }^{1}$ Management of homeless patients is complicated by a lack of identification and of permanent address, ${ }^{2}$ frequent loss to follow-up ${ }^{3}$ and especially higher rates of mental illness and substance use challenges. ${ }^{1,4,5}$

\section{Epidemiology of homelessness in Canada}

Homelessness has become an epidemic in Canada. To give one example of many, more than 4000 people sleep in shelters every night in Toronto. ${ }^{1}$ Experts estimate that the number of homeless individuals in Canada in 2013 numbered approximately $235000 .^{6}$
A further 1 in 7 households in Canada face problems of housing affordability, with housing costs in excess of $30 \%$ of the annual household income. ${ }^{7}$ The demographics of homelessness has also changed significantly in the past 2 decades, from mostly single adult male alcoholics to a diverse population including adolescents, single mothers, the under- and unemployed, the elderly and recent immigrants. ${ }^{4}$

The relationship between homelessness and substance abuse

Substance use is a disorder diagnosed on the basis of recurrent use of a substance leading to impaired control, social impairment and risky use. ${ }^{8}$ It is well established that homelessness and substance use exhibit a bidirectional relationship, with substance use both being more prevalent among people experiencing homelessness ${ }^{4,5}$ and being an independent risk factor for homelessness. ${ }^{9}$ Alcohol use among people experiencing homelessness is estimated to be 6 to 7 times that of the general population. ${ }^{10} \mathrm{~A} 2008$ meta-analysis of alcohol use among homeless adult men found that approximately $38 \%$ had some form of alcohol use disorder. The same meta-analysis found that $24 \%$ of homeless men had a substance use disorder involving one or more substances other than alcohol. ${ }^{11}$

\section{REDUCING HOMELESSNESS THROUGH A HOUSING-FIRST STRATEGY}

Housing-first strategies

A housing-first strategy to reduce homelessness was first conceived and implemented by clinical psychologist Sam Tsemberis in 1992. This program, called Pathways to Housing, was aimed at increasing rehousing rates for homeless individuals with psychiatric disabilities in New York City. ${ }^{12}$

Housing-first strategies mandate the provision of immediate housing, choice and self-determination in rehousing, a focus on recovery, individualized and person-driven supports and social and community integration of the rehoused individual. ${ }^{13}$ Whereas more established treatment-first strategies have stipulated that the individual must be "housing ready" by completing rehabilitation programs aimed at treating substance use and other health challenges, housing-first strategies provide stable, private housing with concurrent, optional rehabilitation programs and other social support

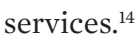

Efficacy of housing-first strategies in reducing homelessness

In one American study of the outcomes of a housing-first intervention in reducing homelessness, researchers found that $57 \%$ to $78 \%$ of people rehoused through housing-first programs remained 
in stable housing after 47 months. ${ }^{15}$ Housing-first strategies also reduce the costs associated with homelessness. In a study by the Mental Health Commission of Canada, a 5-year housing-first strategy resulted in cost savings of between Can $\$ 3.42$ to Can $\$ 9.60$ for every Can $\$ 10.00$ invested. This was achieved through a reduction of utilization of other services by rehoused individuals. ${ }^{13}$

When compared to treatment-first strategies, housing-first strategies have been shown to result in greater housing stability in the first 24 months of starting the program ${ }^{16}$ and overall longer lengths of stay in housing ${ }^{17}$ when compared to treatment-first strategies.

\section{EFFICACY OF HOUSING-FIRST STRATEGIES IN REDUCING SUBSTANCE USE RATES}

Whereas housing-first strategies demonstrate higher overall rates of housing retention, the benefits of a housing-first strategy for rehousing of homeless individuals with a substance use challenge are less clear.

In short-term studies of the efficacy of housing-first strategies in reducing rates of substance use in homeless populations, researchers have found that programs that employ a housing-first strategy actually have greater reductions in rates of alcohol use and relapse into substance use than programs that employ treatment-first strategies. ${ }^{18,19}$ In a review of 29 studies from around the world, Collin et al found that there was a $3 \%$ decrease in overall and peak alcohol consumption in individuals rehoused through a housing-first strategy compared to those rehoused through a treatment-first strategy. ${ }^{18}$ Additionally, Padgett et al showed that participants of a housing-first program were 3.4 times less likely to relapse into substance use than participants of a treatment-first program within the first year. ${ }^{19}$

However, longer-term studies of the efficacy of housing-first programs have largely failed to replicate these promising early results. A 2010 study of the efficacy of housing-first programs across 11 communities across the United States did not identify a significant difference in rates of substance use between housing-first and treatment-first participants after 2 years. ${ }^{17}$ Similarly, a direct comparison between a housing-first intervention and a treatment-first intervention in a homeless population in New York City failed to show improvement in rates of alcohol and substance use with housing-first compared to treatment-first. ${ }^{20}$ The 2014 Canadian At Home/Chez Soi study also found no significant difference between rates of substance use between participants of housing-first versus treatment-first programs, although it also noted an overall improvement in substance use rates amongst both groups. ${ }^{13}$

Despite the lack of long-term improvement in rates of substance use when compared to treatment-first programs, it is important to note that housing-first programs showed equivalent levels of overall reduction in substance use despite not mandating that rehabilitation programs be completed..$^{20}$ In combination with the fact that the overall outcomes are better in housing-first programs than treatment-first programs, it must be concluded that housing-first programs are more effective, or at least not any less effective, than traditional treatment-first programs in rehousing homeless individuals with substance use challenges.

\section{RECOMMENDATIONS FOR REHOUSING OF HOMELESS INDIVIDUALS WITH SUBSTANCE USE CHALLENGES}

Stably rehousing homeless individuals with substance use challenges is a complex task that demands an interdisciplinary case management team. In studies of best practices in the management of homelessness, researchers have identified 6 features of successful case management strategies: client identification and outreach, assessment of needs, planning of treatment and services, linking the client to necessary resources, monitoring progress and client advocacy. Existing studies on the management of this sub-population of patients have emphasized adoption of assertive community treatment (ACT) teams. ${ }^{21}$

The ACT team model was developed for treatment of individuals with severe mental illness, and includes a physician or nurse experienced in the diagnosis and management of mental illness and substance use challenges, a psychiatrist to manage symptoms of mental illness and monitor effectiveness and adherence to medications, social services to help with social reintegration (including support for obtaining education and/or employment, a personal support worker to assist with activities of daily living and a peer specialist who acts as a counselor to facilitate the treatment process). ${ }^{22}$

Based on the findings presented in this review, we propose that the following guidelines be followed when considering the approach to rehouse homeless individuals with substance use challenges:

1. A housing-first strategy is equally viable to a treatment-first strategy in rehousing of homeless individuals with substance use challenges. ${ }^{20}$

2. Rehoused individuals with known substance use challenges should be identified and support should be offered in the form of an interdisciplinary team of healthcare providers, including a physician, nurse, psychiatrist, personal support worker and peer specialist; care should be provided using a client-centred model and should include a case manager who can coordinate the team's actions. ${ }^{21,22}$

3. The interdisciplinary team providing care for rehoused individuals with known substance use challenges should continue working with those individuals for a period of several years to ensure long-term retention in stable housing. ${ }^{17,20}$

\section{CONCLUSION}

The implementation of housing-first strategies has the potential to make a real positive impact on homelessness in Canada. In this review we examined the efficacy of housing-first strategies in rehousing individuals with substance use challenges and in reducing rates of substance use among rehoused individuals. Although we found an initial improvement in reduction of substance use in individuals rehoused through a housing-first strategy compared to those rehoused through a treatment-first strategy, current available evidence does not support the existence of any significant longterm reduction in rates of substance use beyond what is achieved with treatment-first strategies. However, we found that housing-first strategies provided a number of other advantages over 
treatment-first strategies. We conclude that housing-first strategies are slightly better than treatment-first strategies in rehousing those homeless individuals with a substance use challenge.

\section{REFERENCES}

4. Hwang SW. Mental illness and mortality among homeless people. Acta Psychiatr Scand. 2001;103:81-2.

5. Khandor E, Mason K, Chambers C, Rossiter K, Cowan L, Hwang SW. Access to primary health care among homeless adults in Toronto, Canada: results from the Street Health survey. Open Med. 2011;5(2):e94.

6. Conwell DS, Mosher A, Khan A, Tapy J, Sandman L, Vernon A, et al. Factors associated with loss to follow-up in a large tuberculosis treatment trial (TBTC study 22). Contemp Clin Trials. 2007 May;28(3):28896.

7. Hwang SW. Homelessness and health. CMAJ. 2001;164(1):229-33.

8. Turnbull JS, Muckle W, Masters C. Homelessness and health. CMAJ. 2007 Oct;177(9):1065-6.

9. Gaetz S, Gulliver T, Richter T. The state of homelessness in Canada 2014. Toronto: The Homeless Hub Press; 2014. 73p.

10. Laird G. SHELTER: homelessness in a growth economy: Canada's 21st century paradox. Calgary (AB): Sheldon Chumir Foundation for Ethics in Leadership; 2007 p.

11. American Psychiatric Association. Diagnostic and statistical manual of mental disorders. 3rd ed. Washington: American Psychiatric Association; 2013.

12. Dixon L. Dual diagnosis of substance abuse in schizophrenia: prevalence and impact on outcomes. Schizophrenia Res. 1999;35:S93-100.

13. Webb GR, Redman S, Gibberd RW, Sanson-Fisher RW. The reliability and stability of a quantity-frequency method and a diary method of measuring alcohol consumption. Drug Alcohol Depend. 1991 May;27(3):223-31.

14. Fazel S, Khosla V, Doll H, Geddes J. The prevalence of mental disorders among the homeless in Western countries: systematic review and meta-regression analysis. PLoS Med. 2008 Dec;5(12):1670-81.

15. Tsemberis S, Eisenberg RF. Pathways to Housing: supported housing for street-dwelling homeless individuals with psychiatric disabilities. Psychiatr Serv. 2000 Apr;51(4):487-93.

16. Goering P, Veldhuizen S, Watson A, Adair C, Kopp B, Latimer E, et al. National At Home/Chez Soi final report. Calgary (AB): Mental Health Commission of Canada.

17. Pleace N, Bretherton J. The case for housing first in the European Union: a critical evaluation of concerns about effectiveness. Eur J Homelessness. 2013 Dec;7(2):21-41.

18. Stefancic A, Tsemberis S. Housing first for long-term shelter dwellers with psychiatric disabilities in a suburban county: a four-year study of housing access and retention. J Primary Prevent. 2007

19. Tsemberis S, Gulcur L, Nakae M. Housing first, consumer choice, and harm reduction for homeless individuals with a dual diagnosis. Am J Public Health. 2004 Apr;94(4):651-6.

20. Tsai J, Mares AS, Rosenheck RA. A multi-site comparison of supported housing for chronically homeless adults: "housing first" versus "residential treatment first.” Psychol Serv. 2010;7(4):219-32.

21. Collins SE, Malone DK, Clifasefi SL, Ginzier JA, Garner MD, Burlingham B, et al. Project-based housing first for chronically homeless individuals with alcohol problems: within-subjects analyses of 2-year alcohol trajectories. Am J Public Health. 2012 Mar;102(3):511-9.

22. Padgett DK, Stanhope V, Henwood BF, Stefancic A. Substance use outcomes among homeless clients with serious mental illness: comparing housing first with treatment first programs. Community Ment Health
J. 2011 Apr;47(2):227-32.

23. Padgett DK, Gulcur L, Tsemberis S. Housing first services for people who are homeless with co-occurring serious mental illness and substance abuse. Res Soc Work. 2006 Jan;16(1):74-83.

24. Morse G. A review of case management for people who are homeless: implications for practice, policy, and research. In: Fosburg LB, Dennis DL, editors. Practical Lessons: The 1998 National Symposium.

25. Bond GR, Drake RE, Mueser KT, Latimer E. Assertive community treatment for people with severe mental illness: critical ingredients and impacts on patients. Dis Manage Health Outcomes. 2001 Mar;9(3):141-59. 